\title{
Dificuldades Alimentares na Primeira Infância: Uma Revisão Sistemática
}

\section{Feeding Difficulties in Infancy: A Systematic Review}

\section{Dificultades Alimentares em la Primera Infancia: Una Revisión Sistemática}

\author{
Patrícia Wolff Müller* \\ Universidade do Vale do Rio dos Sinos - UNISINOS, São Leopoldo, Rio Grande do \\ Sul, Brasil
}

\author{
Viviane Salazar** \\ Universidade do Vale do Rio dos Sinos - UNISINOS, São Leopoldo, Rio Grande do \\ Sul, Brasil
}

\section{Tagma Marina Schneider Donelli***}

Universidade do Vale do Rio dos Sinos - UNISINOS, São Leopoldo, Rio Grande do Sul, Brasil

\begin{abstract}
RESUMO
Dificuldades no âmbito da alimentação podem incluir alterações na qualidade ou na quantidade da alimentação, dificuldades com alimentos específicos que são introduzidos durante o primeiro ano de vida da criança, além de afetarem na maneira como mães e bebês se envolve, pois a relação de alimentação entre a mãe e o bebê é influenciada, desde o início tanto por fatores fisiológicos como por forças interacionais em múltiplos níveis. Considerando-se a importância da alimentação da criança para a promoção de sua saúde e para a relação entre mãe e filho, este artigo visa realizar uma revisão sistemática da literatura internacional especializada sobre as dificuldades alimentares na primeira infância, de itens publicados nos últimos cinco anos, nas bases de dados Ebsco Host (Academic Search e Medline), Pubmed/Medline, Psychinfo, Science Direct, Scopus, BVS e Scielo. Foram selecionados 24 artigos da área da Psicologia. Identificou-se que o método quantitativo com características prospectivas e longitudinais foi 0 mais utilizado entre os estudos. Além disso, o continente europeu é responsável pela maioria das publicações. Percebeu-se um crescimento de estudos sobre a questão alimentar na infância a partir do ano de 2010 e os participantes foram, em sua maior parte, mães e duplas de mães e bebês. Dentre os objetivos, destacou-se o propósito de pesquisar a relação entre os problemas de alimentação e as práticas e estilos parentais, enquanto que os resultados mostraram que o comportamento materno possui influência na alimentação, esta podendo ser usada como uma forma de controle e de conforto.
\end{abstract}

Palavras-chave: dificuldades alimentares, alimentação, infância, relação mãe-bebê, revisão da literatura. 


\begin{abstract}
Difficulties in the context of food may include changes in the quality or quantity of food as well as difficulties with specific foods that are introduced during the first year of the child's life, as well as affecting the way mothers and babies engage because the feeding relationship between mother and baby is influenced from the outset by both physiological factors and interactional forces at multiple levels. Considering the importance of child nutrition for the promotion of their health and mother-baby relation, this article aims to conduct a systematic review of the international literature on specialized feeding difficulties in early childhood, of items published in the last five years, Ebsco Host databases (Academic Search and Medline), Pubmed/Medline, Psychinfo, Science Direct, Scopus, VHL and Scielo. 24 articles were selected from the field of Psychology. It was identified that the quantitative method with prospective and longitudinal features was the most used among the studies. In addition, the European continent is responsible for the majority of publications. It was realized a growth of studies on the food issue in childhood from the year 2010 and the participants were, for the most part, mothers and pairs of mothers and babies. Among the goals, said the purpose of researching the relationship between the problems of feeding and the parenting styles and practices, while the results showed that maternal behavior has an influence on feeding, this can be used as a form of control and comfort.
\end{abstract}

Keywords: feeding difficulties, feeding, childhood, mother-child relationship, the literature review.

\title{
RESUMEN
}

Dificultades bajo el poder pueden incluir cambios en la calidad o cantidad de los alimentos, así como las dificultades con los alimentos específicos que se introducen durante el primer año de vida del niño, así como afectar la forma en que las madres y los bebés está involucrado porque la relación de poder entre la madre y el bebé se ve influida desde el principio tanto por factores fisiológicos tales como las fuerzas de interacción en múltiples niveles. Teniendo en cuenta la importancia de la nutrición para la promoción de la salud y para la relacción entre madre y hijo, este artículo pretende realizar una revisión sistemática de la literatura internacional sobre las dificultades alimentares en la primera infancia, de los artículos publicados en los últimos cinco años, las bases de datos Ebsco Host (Academic Search y Medline), Pubmed/Medline, Psychinfo, Science Direct, Scopus, BVS y Scielo. Se seleccionaron 24 artículos del campo de la psicología. Se identificó que el método cuantitativo con características prospectivas y longitudinales es el más utilizado entre los estudios. Además, el continente europeo es responsable de la mayoría de las publicaciones. Se observó un crecimiento de estudios sobre el tema de alimentos en la infancia desde el año 2010 y los participantes eran, para la mayor parte, las madres y los pares de madres y bebés. Entre los objetivos, dijo que el propósito de investigar la relación entre los problemas de alimentación y los estilos de crianza y las prácticas, mientras que los resultados demostraron que el comportamiento materno tiene una influencia sobre la alimentación, esto puede usarse como una forma de control y comodidad.

Palabras clave: alimentación relación madre-recién nacido, infancia, dificultades, alimentos, revisión de la literatura. 


\section{I ntrodução}

A primeira infância é o período compreendido desde a concepção até o sexto ano de vida (Schneider \& Ramires, 2007), e nessa época as perturbações alimentares são situações clínicas muito frequentes que, em geral, provocam nos pais grande preocupação que repercute quase sempre na relação com a criança. Vão desde flutuações do apetite, transitórias e relacionadas a acontecimentos tênues, como alterações no cotidiano ou no ambiente, entrada na creche, nascimento de irmãos, até situações de recusa alimentar graves que põem em risco a própria vida da criança. Estes comportamentos podem ser acompanhados ou não do aparecimento de comportamentos anômalos e bizarros (Gonçalves \& Rodrigues, 1998). Segundo Carvalho, Lima e Martins (2013a), a alimentação é um dos cuidados essenciais que se tem com o bebê, e as experiências alimentares iniciais serão organizadoras e estruturantes da personalidade deste sujeito em formação. Com isso, a alimentação torna-se uma questão de relação da mãe com o filho, em que se põe em prática a relação de amor entre dois seres humanos (Winnicott, 1965/1982).

Sendo assim, a alimentação pode ser considerada como a principal via de troca e comunicação entre a criança e seus cuidadores, e as dificuldades existentes na relação mãe-bebê facilmente desenvolvem sintomas ligados à esfera alimentar. Ou seja: quando as operações materna e paterna funcionarem de maneira estruturante para o bebê, - esperado será que as trocas alimentares e todos os demais processos de troca se deem de uma forma que não gerem fixações patológicas (Carvalho et al., 2013a). Contudo, a ansiedade e a frustração dos pais e, sobretudo da mãe, causada pela recusa alimentar da criança, e a associação que estabelecem entre a recusa alimentar dos filhos e os seus sentimentos de insuficiência maternal, provocam uma baixa da autoestima e levam a que tenham atitudes que agravam as dificuldades da criança (Gonçalves \& Rodrigues, 1998). Adami-Lauand e Ribeiro (2011) conjeturam sobre o papel da família nos transtornos alimentares, e, para elas, é através da família que as transmissões das regras e hábitos culturais encontram espaço para coabitarem com vínculos afetivos construídos pela interação entre seus membros e suas histórias. A alimentação torna-se a via pela qual essas regras e vínculos podem ser transmitidos pelas gerações.

Em termos de classificação diagnóstica se faz necessário diferenciar os termos transtornos alimentares e transtornos da alimentação. Segundo Gusmão (2002), transtornos da alimentação, ou feeding disorder, geralmente é usado para dar destaque à natureza dual ou diádica dos problemas alimentares em bebês ou crianças pequenas. Neste caso, há uma perturbação no ato de dar de comer, enquanto 
que os transtornos alimentares, ou eating disorders, se referem a perturbações no ato de comer.

O Manual Diagnóstico e Estatístico de Transtornos Mentais [DSM-V] (American Psychiatric Association, 2013) os transtornos alimentares e os transtornos da alimentação são caracterizados por uma perturbação persistente de comer, ou estão relacionados ao comportamento de comer, que resulta na alteração do consumo ou da absorção de alimentos, e que prejudica seriamente a saúde física ou o funcionamento psicossocial. Os critérios diagnósticos para o transtorno de ruminação, transtorno de ingestão de alimentos esquiva / restritiva, anorexia nervosa, bulimia nervosa e transtorno como resultado da compulsão alimentar periódica estão organizados em um esquema de classificação que é mutuamente exclusiva, de modo que durante um único episódio, apenas um desses diagnósticos pode ser atribuído. Apesar de uma série de características psicológicas e comportamentais comuns, os distúrbios diferem substancialmente em necessidades, curso, evolução e tratamento clínico. O diagnóstico de pica, no entanto, poderá ser aplicado, na presença de qualquer outra alimentação e distúrbios alimentares.

Antes de se utilizar de qualquer ferramenta de classificação diagnóstica, é importante que se conheça como é o desenvolvimento da criança na faixa etária em que ela se encontra, fazendo uma compreensão do seu funcionamento dentro da dinâmica familiar (Pinto, 2004). O Zero to Three (DC: 0-3R, 2005) procura fazer uma classificação sistemática, desenvolvimental, de saúde mental e dificuldades no desenvolvimento nos primeiros quatro anos de vida. Considera como transtorno do comportamento alimentar a criança que tem dificuldade em estabelecer padrões regulares de alimentação, quando essa não consegue regular sua alimentação de acordo com sensações fisiológicas de fome ou de plenitude. De acordo com o DC: $0-3$, há seis subcategorias de transtornos que são: 1) Estado regulatório; 2) Reciprocidade entre cuidador e bebê; 3) Anorexia infantil; 4) Aversão sensória a comida; 5) Transtorno da alimentação associado com uma condição médica atual 6) Transtorno da alimentação associada com trato gastrintestinal.

Chatoor (2002) desenvolveu uma classificação diagnóstica que é a seguinte: a) Transtorno da alimentação do estado regulatório (ocorre durante o período de recém-nascido); b) Transtorno da alimentação de reciprocidade (ocorre entre dois e seis meses de idade); c) Anorexia infantil (ocorre durante a transição de alimentação dada por outra pessoa à criança se alimentando sozinha); d) Aversão sensorial alimentar (ocorre durante a introdução da alimentação de bebê ou alimentação da família); e) Transtorno da alimentação associado com condições médicas atuais; f) Transtorno alimentar pós-trauma. As duas últimas classificações podem ocorrer em qualquer idade. Bryant-Waugh, Markham, Kreipe e Walsh (2010) sugerem pensar o 
transtorno dentro de um contexto levando em conta características das crianças e dos pais. Contudo, as causas comportamentais são mais difíceis de serem tratadas, pois são determinadas em sua maior parte por erros de concepção ou conduta dos pais (Madeira \& Aquino, 2003).

Segundo Madeira e Aquino (2003), as dificuldades alimentares e de sono são comuns em pediatria e essas podem fazer parte das fases do desenvolvimento normal da criança rumo à sua independência. As autoras destacam ainda que o desconhecimento das necessidades nutricionais reais das crianças e o desconhecimento das etapas do desenvolvimento emocional e comportamental por parte dos pais seriam possíveis causas para as dificuldades alimentares. Nicholls e Bryant-Waugh (2009) referem que tais dificuldades podem ocorrer em crianças com desenvolvimento normal, mas também naquelas com condições médicas crônicas ou com transtornos no desenvolvimento. As causas podem ser multifatoriais, no entanto, tentativas têm sido feitas para classificar dificuldades de alimentação que surgem principalmente de dificuldades psicológicas e de relacionamento, e não como resultado de problemas orgânicos ou de desenvolvimento. O DSM - V (2013) ressalta o diagnóstico diferencial. Condições médicas como doenças gastrointestinais, alergias e intolerâncias alimentares e neoplasias ocultas, comorbidades subjacentes podem interferir na alimentação e no comer. Para Brazelton (2002), somente aos quatro anos é que a criança talvez esteja pronta para comer de tudo. Até chegar nessa etapa, a dieta e ingestão dos alimentos sofrerá variações. Na área da alimentação, comportamentos em busca da independência e o negativismo fazem parte do desenvolvimento até os quatro anos. No entanto, os pais sentem que a responsabilidade de alimentar o filho é deles. Assim, permitir que o filho expresse suas preferências dá-Ihes uma sensação de vazio, de não estarem o cuidando como deveriam.

Do nascimento até os três anos de idade é considerado um período em que aquisições fundamentais para a constituição do sujeito estão sendo sedimentadas. É nesse período que o psiquismo e as funções mentais estão começando a se estruturar. Sob esse aspecto, as inconstâncias desse tempo da constituição psíquica são determinantes para o desenvolvimento da criança (Carvalho et al., 2013). Sendo assim, estudos que abordam a primeira infância são fundamentais, pois essa é uma etapa crucial para o desenvolvimento emocional, físico e neurológico. Winnicott (1965/1982) considera importante estudar o desenvolvimento emocional em suas primeiras fases. Para ele, muitas vezes é possível detectar e diagnosticar distúrbios emocionais ainda na infância, até mesmo no primeiro ano de vida. Micali, Smirnoff, Stahl e Treasure (2011) ressaltam a importância dos resultados das pesquisas no âmbito dos distúrbios alimentares como forma de prevenção e intervenção precoce em 
relação às dificuldades de alimentação infantis, e também para futuras pesquisas na área.

Ponderando que a questão alimentar é de fundamental importância para a relação entre mãe e bebê, que é essencial para o processo de comunicação entre a dupla e que serve de modelo para as relações interpessoais que virão. Com isso, as dificuldades alimentares podem ser consideradas esperadas, de acordo com a fase do desenvolvimento em que a criança se encontra, mas também podendo representar algo mais sério como um transtorno. Buscou-se realizar um levantamento bibliográfico com o intuito de verificar o que está sendo publicado na literatura científica, pois estudos e intervenções na primeira infância são de grande valor para pensar tanto em desenvolvimento de enfermidades na área da alimentação quanto em possíveis intervenções pais e bebês com sintomas alimentares. Assim, este trabalho tem como objetivo realizar uma revisão sistemática da literatura especializada sobre as dificuldades alimentares em crianças.

\section{Método}

Foi realizado levantamento bibliográfico nas bases de dados internacionais Ebsco Host (Academic Search e Medline), Pubmed, Psychinfo, Science Direct, e Scopus. Utilizou-se os seguintes descritores: feeding difficulties, feeding disorder e feeding problems, juntamente com as palavras child e infancy, a fim de localizar o maior número de artigos publicados sobre o tema. A pesquisa abrangeu os últimos cinco anos tendo sido feitos cruzamentos entre todos esses descritores com o intuito de se encontrar o máximo de produção científica na temática estudada.

Para a seleção dos estudos, foram utilizados os seguintes critérios de inclusão: 1) artigos empíricos; 2) disponibilização de texto completo; 3) publicados em língua inglesa, espanhola e portuguesa; 4) da área da Psicologia; 5) ter como participantes das pesquisas crianças menores de seis anos e/ou seus pais. Para exclusão, utilizaram-se os critérios: 1) duplicados; 2) não contemplavam os descritores; 3) participantes acometidos de uma doença crônica, síndromes e outros transtornos do desenvolvimento.

A figura a seguir apresenta um fluxograma da metodologia aplicada na busca dos artigos: 


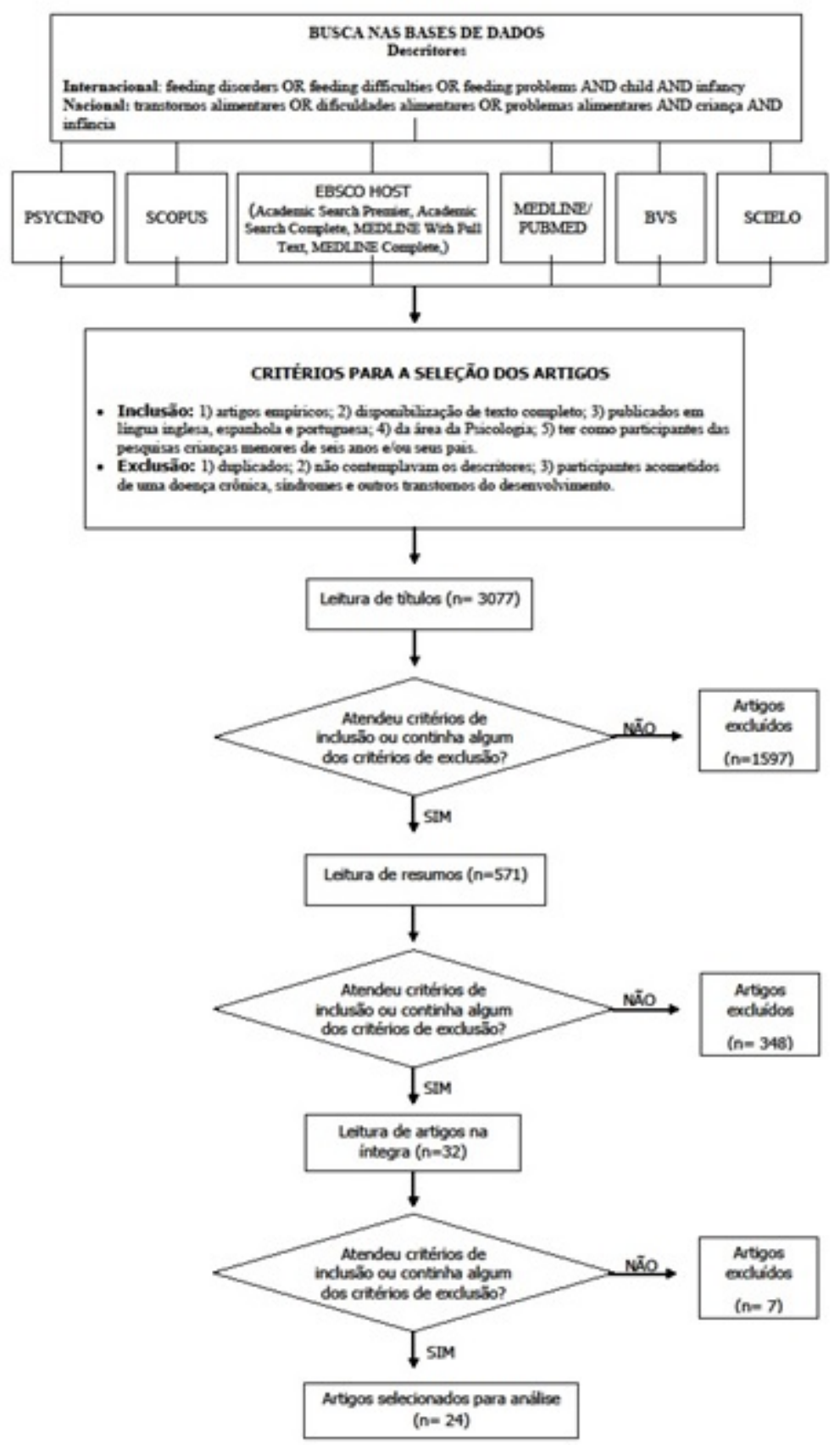

Figura 1: Fluxograma da Revisão Sistemática

\section{Resultados e Discussão}

A partir da pesquisa por estudos nas bases eletrônicas consultadas, aplicando os descritores, obteve-se um resultado de 3.077 artigos. Após a leitura dos títulos, resumos e utilizando os critérios de inclusão e exclusão, obteve-se 24 artigos (Ammaniti et al., 2012; Atzaba-Poria et al., 2012; Blisset et al., 2011; Carvalho et al., 2013b; Dovey et al., 2013; Farrow, Galloway, \& Fraser, 2009; Forestell \& Mennella, 2012; Gaffney, Kitsantas, Brito, \& Swamidoss, 2014); 
Hoffman et al., 2013; Jones \& Bryant-Waugh, 2012; Koh, Scott, Woodman, Kim, Daniels \& Magarey, 2014; Li, Scanlon, May, Rose, \& Birch, 2014; Mc Dermott et al., 2010; McMeekin et al., 2013; Micali et al., 2011; Östberg \& Hagelin, 2011; Owen, Ziebel, Lessard, Churcher, Bourget, \& Villeneuve, 2012; Powell, Farrow, \& Meyer, 2011; Reba-Harrelson et al., 2010; Richter \& Reck, 2013; Rigal et al., 2012; Schmid et al., 2010; Tambelli, Odorisio, \& Lucarelli, 2014; Thompson \& Bentley, 2013) que foram analisados na íntegra, com base nos eixos descritos na metodologia.

\section{Local de origem dos estudos}

Percebe-se que a Europa tem se focado nos estudos sobre a questão alimentar em crianças (J ones \& Bryant-Waugh, 2012; Dovey, Jordan, Adridge, \& Martin, 2013; Powell, Farrow, \& Meyer, 2011; Micali, Smirnoff, Stahl, \& Treasure, 2011; Blisset, Meyer, \& Haycraft, 2011; Richter, \& Reck, 2013; Tambelli et al., 2014; Östberg \& Hagelin, 2011; Rigal et al., 2012). Os países contribuintes desse continente foram Alemanha, França, Itália, Suécia e Inglaterra, a qual teve a maior quantidade de estudos. Na América do Norte, tiveram-se artigos tanto Estados Unidos (Forestell \& Mennella, 2012; Gaffney et al., 2014; Hoffmann et al., 2013; Li, et al., 2014; Thompson \& Bentley, 2013) quanto Canadá (Owen et al., 2012). Na América do Sul, encontrou-se um estudo do Brasil (Carvalho, et al., 2013b), bem como na Ásia teve um estudo de Israel (Atzaba-Poria et al., 2012). $\mathrm{Na}$ Oceania, a Austrália publicou alguns estudos sobre o tema em discussão (Koh et al., 2014; Mc Dermott et al., 2010; McMeekin et al., 2013). Houve também estudos em parceria com autores de nacionalidades diferentes como os de Ammaniti et al. (2012) (Itália e Inglaterra), Farrow e Meyer (2011) (Estados Unidos e Reino Unido), Reba-Harrelson et al. (2010) (Estados Unidos e Noruega) e ainda Schmid et al. (2010) (Suíça e Inglaterra).

$\mathrm{Na}$ figura 2 pode-se perceber a quantidade de artigos publicados em relação aos continentes em que foram escritos. 


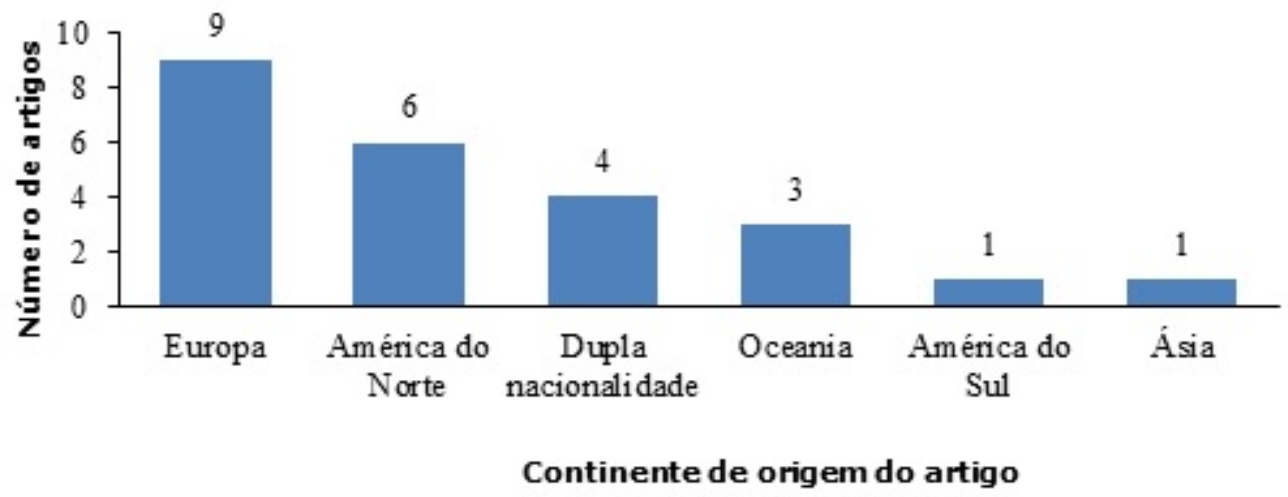

Figura 2: Relação dos artigos com base no continente em que foi escrito.

\section{Ano de publicação}

Do período analisado (2009 a 2014), a publicação de artigos na área de dificuldades alimentares na primeira infância teve um aumento após 2010, sendo 2013 o ano de maior publicação, conforme pode ser visto na figura 3.

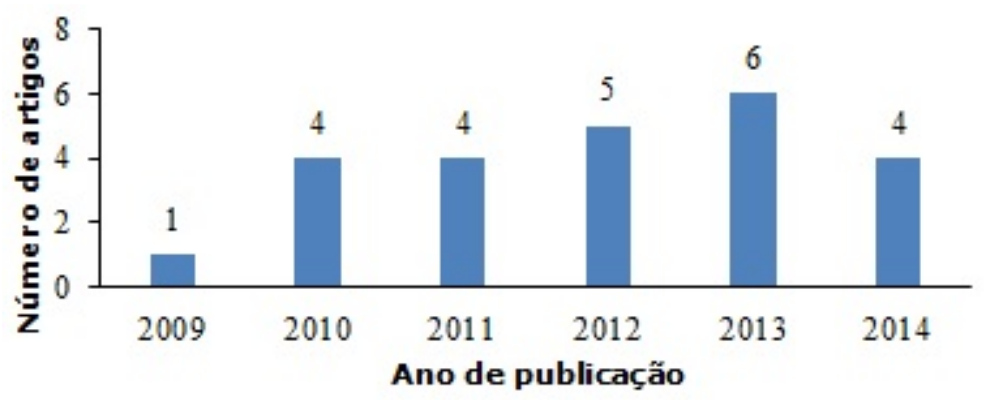

Figura 3: Ano de publicação dos artigos

\section{Metodologia empregada nos estudos}

Na figura 4 é apresentada a distribuição dos artigos analisados quanto à sua metodologia.

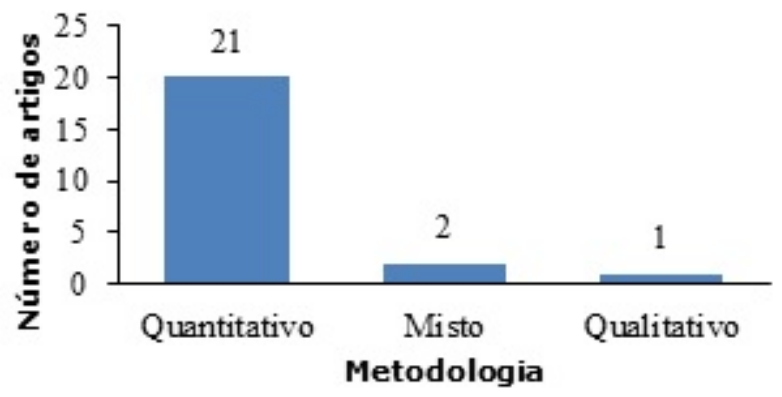

Figura 4: Relação entre os artigos analisados e a metodologia empregada 
Analisando o gráfico acima, pode-se dizer que há uma preocupação nos estudos quantitativos - os quais eram predominantemente prospectivos ou longitudinais com características epidemiológicas em mapear as dificuldades alimentares e se estas permanecem e como se manifestam com o passar do tempo. Nesses estudos, o tema da alimentação foi considerado uma questão de saúde pública, e esses trabalhos, em função do seu delineamento, podem contribuir para estratégias em saúde e políticas públicas. No entanto, considera-se importante a realização de estudos qualitativos, pois estes proporcionam uma investigação aprofundada e fornecem detalhes sobre os sintomas e de que forma eles se manifestam, adotando uma postura compreensiva do fenômeno.

\section{Participantes}

Identificou-se que grande parte dos estudos analisados teve como participantes as mães (Blisset et al., 2011; Jones \& Bryant-Waugh, 2012; McMeekin et al., 2013; Powell, Farrow \& Meyer, 2011; Rigal et al., 2012; Schmid et al., 2010) e a dupla mãe e bebê (Ammaniti et al., 2012; Carvalho, et al., 2013b; Gaffney et al., 2014; Hoffmann et al., 2013; Koh et al. (2014); McMeekin et al., 2013; Micali et al., 2011; Reba-Harrelson et al., 2010; Richter \& Reck, 2013; Thompson \& Bentley, 2013), mas também famílias (Dovey et al., 2013; Maldonado-Duran et al., 2011; Östberg \& Hagelin, 2011; Owen et al., 2012). Isso demonstra que é importante considerar o contexto onde a criança está inserida e a relação desta com as pessoas mais próximas, pois isto pode ter influência nas dificuldades alimentares.

A idade das crianças participantes variou de acordo com cada estudo, mas nota-se que nunca é cedo para averiguar as dificuldades alimentares e investigar isso no relacionamento entre mãe e bebê, principalmente no contexto da refeição. No trabalho de Micali et al., (2011) participaram gestantes e bebês de até oito meses de idade. Já McDermott et al. (2010) realizaram um estudo em que o intervalo de idade dos participantes abrangeu desde o nascimento até os 14 anos de idade.

\section{Objetivos}

Os objetivos dos artigos analisados foram agrupados conforme a sua semelhança e estão apresentados na tabela 1. 
Tabela 1

Objetivos dos artigos analisados

\begin{tabular}{|c|c|c|}
\hline Objetivo & $\begin{array}{l}\text { Numero de } \\
\text { Artigos }\end{array}$ & Autor(es) \\
\hline $\begin{array}{l}\text { Analissu se ha relaçào entre os problemas de } \\
\text { almentaçio e as praticas e estalos purentus }\end{array}$ & 6 & $\begin{array}{l}\text { Busset et al. } 2011 \text {; } \\
\text { Carvalho. Lma. Martuns. } 20136 . \\
\text { Hoffmarn et al. } 2013 \text { : } \\
\text { Powell et al. 2011. } \\
\text { Peba-Harrelsco et al. 2010: } \\
\text { Pichter \& Red. 2013 }\end{array}$ \\
\hline $\begin{array}{l}\text { Verificar a percepsio dos pass solre a prauca almentar } \\
\text { en diferentes idades }\end{array}$ & 2 & $\begin{array}{l}\text { McDermot et al. } 2010 . \\
\text { Mcleckin et al. } 2013\end{array}$ \\
\hline Desenvolver e avabar os resultados de uma mtervencià & 2 & $\begin{array}{l}\text { Jones \& Bryant-Wangh. 2012: } \\
\text { Oren et al. } 2012\end{array}$ \\
\hline Validar um motrumento & 2 & $\begin{array}{l}\text { Dovey et al . 2013: } \\
\text { Pigal et al. } 2012\end{array}$ \\
\hline $\begin{array}{l}\text { Examinar os padtòes de almentaçà das crianças e o } \\
\text { seu fincucoumento emocional e couportamental }\end{array}$ & 2 & $\begin{array}{l}\text { Ammanut. 2012; } \\
\text { Forestell \& Mennella } 2012\end{array}$ \\
\hline $\begin{array}{l}\text { Investigar interaçòes entre pais e filhos com distirtios } \\
\text { almentares }\end{array}$ & 2 & $\begin{array}{l}\text { Atzaba-Pona et al, } 2010 \text {; } \\
\text { McKechin et al. } 2013\end{array}$ \\
\hline $\begin{array}{l}\text { Pealizar grupos distintos cam cranças para canhecer o } \\
\text { comportamento almentar e emocional da amostra }\end{array}$ & 1 & Ammunitiet al, 2012 \\
\hline $\begin{array}{l}\text { Acompanhar cruaças cam problemas precoces de } \\
\text { almentaçio e ou dormir e avenguar se aunda tnhum } \propto s \\
\text { mesmos problemas apos seis anos }\end{array}$ & 1 & Ostberg \& Hagelin 2011 \\
\hline $\begin{array}{l}\text { Investagar o papel da ansiedade e depressio matema na } \\
\text { mediaça do risco para dificuldades alumentares }\end{array}$ & 1 & Macali et al. 2011 \\
\hline 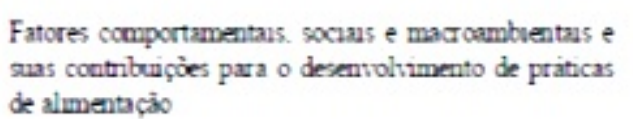 & 1 & Thompson e Bentley (2013) \\
\hline
\end{tabular}

\section{I nstrumentos}

Os instrumentos utilizados nos estudos quantitativos variaram desde escalas e questionários estruturados (Blisset et al., 2011; Hoffman et al., 2013; Forestell \& Mennella, 2012; McMeekin et al., 2013; Micali et al., 2011; Richter \& Reck, 2013; Östberg \& Hagelin, 2011; Owen et al., 2012; Rigal et al., 2012; Schmid et al., 2010). Percebeu-se que para avaliar a questão alimentar, as ferramentas mais usadas foram os questionários padronizados como o Child Behavior Checklist (CBCL) e Comprehensive Feeding Practices Questionnaire (CFPQ), além de vídeo da refeição (Atzaba-Poria et al., 2010; Forestell \& Mennella, 2012; Hoffmann et al., 2013). 


\section{Resultados}

Assim como os objetivos, os resultados encontrados nos estudos foram variados. Os achados de Blisset et al. (2011), Forestell \& Mennella (2012), Micali et al., (2011), Powell et al. (2011) e Rigal et al., (2012) mostram que os comportamentos maternos em relação às dificuldades alimentares da criança tendem a aumentar e influenciar na quantidade de comida ingerida e também nos transtornos alimentares. Práticas como pressão para a criança comer tiveram influência no comportamento de evitação de alimentos. Powell et al. (2011) e McMeekin et al. (2013) consideraram que o alimento pode ser usado tanto como uma forma de controle como uma forma de conforto por haver dificuldade, por parte dos pais, em perceber os sinais de fome por parte da criança. Thompson e Bentley (2013) consideram que a obesidade na infância é um problema crescente de saúde pública, especialmente entre as crianças das minorias. As disparidades na prevalência do excesso de peso persistem e aumentam com a idade. Com isso, torna-se importante identificar os fatores que contribuem para o excesso de ganho de peso precoce. As características estruturais e sociais de práticas de alimentação sólida têm o potencial para moldar o desenvolvimento da obesidade na primeira infância. Os resultados encontrados pelos autores sugerem que a introdução aos alimentos sólidos ocorre na idade e de maneira inadequada, assim como uma má qualidade da dieta. Além disso, a amamentação está associada a uma menor chance de alimentação indevida de sólidos e líquidos. Os autores consideram que intervenções focadas a melhorar as características físicas e sociais do ambiente podem ser extremamente importantes para reverter este desenvolvimento.

Os resultados encontrados por Ammaniti et al. (2012) são de grande importância, pois perceberam uma melhora parcial no estado nutricional das crianças com anorexia infantil. Houve correlações significativas entre problemas alimentares das crianças e suas dificuldades emocionais e maior sofrimento emocional de suas mães e distúrbios na alimentação. Tanto Ammaniti et al. (2012) como Östberg e Hagelin (2011), McDermott et al. (2010) e Schmid et al. (2010) verificaram que os problemas alimentares ou regulatórios persistiram ao longo do período em que a pesquisa foi realizada. Problemas psicossociais e de internalização das crianças foram comuns nos estudos de Ammaniti et al. (2012) como Östberg e Hagelin (2011) e Schmid et al. (2010). Carvalho, Lima e Martins (2013b), perceberam que a desnutrição pode estar relacionada a uma dificuldade em renunciar ao objeto seio e realizar substituições para outros objetos/alimentos. Sendo assim, torna-se importante levar em consideração os fatores psíquicos relacionados ao âmbito da relação mãe-criança. 
Os autores que propuseram intervenções obtiveram resultados significativos. A proposta de Jones e Bryant-Waugh (2012) foi de realizar grupos com mães de crianças com problemas alimentares. Apesar de não ter havido resultados significativos nos níveis de humor e estresse das mães, o grupo oportunizou a melhora da percepção materna em relação à alimentação infantil e também reduziu a sensação de isolamento e a auto culpa. Já a intervenção proposta por Atzaba-Poria et al. (2010) propôs analisar a interação entre pais e filhos em dois momentos diferentes: durante a refeição e durante uma brincadeira. Os resultados mostraram que tanto as interações mãe-filho quanto as interações pai-filho são menos positivas nas famílias transtorno da alimentação do que as do grupo de comparação. Além disso, no grupo de transtorno da alimentação, as mães apresentaram maior sensibilidade do que os pais, mas essa diferença é moderada pelo envolvimento paterno.

\section{Considerações Finais}

Nesta revisão da literatura científica internacional, foram encontrados vinte e quatro artigos sobre as dificuldades alimentares em crianças, o que pode ser considerado um resultado discreto, tendo em vista a relevância da temática. O número inicial de 3.077 itens obtidos com os descritores eleitos demonstra que o assunto vem sendo investigado, principalmente nos últimos anos. Como a maioria dos trabalhos excluídos da análise tratava da alimentação vinculada a condições médicas e orgânicas, ou associadas a problemas no desenvolvimento infantil, considera-se importante investigar a temática também quando esta não está atrelada a situações extremas vividas na infância. É importante atentar que a alimentação da criança é considerada um aspecto fundamental para a promoção de sua saúde, ultrapassa o âmbito biológico e inclui também uma dimensão psicológica que perpassa as primeiras relações entre a mãe e a criança, sendo esta entendida como uma relação fundamental que influencia toda a sua constituição subjetiva.

Entre 2010 e 2013 houve maior número de produções internacionais abordando as dificuldades alimentares em crianças. A produção tem se mantido estável. Nota-se que as dificuldades alimentares têm sido tema constante de estudos, principalmente na Europa e Estados Unidos. Ponderando que as questões das dificuldades na área da alimentação são frequentes na prática pediátrica e uma das queixas principais também na clínica psicológica com bebês e crianças pequenas, torna-se importante desenvolver estudos nacionais sobre o tema na área da Psicologia, principalmente contemplando a primeira infância, etapa decisiva do desenvolvimento infantil. 
Apesar de não haver um consenso sobre a origem das dificuldades alimentares em crianças nos estudos revisados, a produção científica analisada mostra que é de grande relevância as relações familiares e o ambiente em que a criança está inserida, a fim de compreender a origem de suas dificuldades. Além disso, percebeu-se uma preocupação em averiguar como estes problemas se apresentam ao longo do desenvolvimento da criança, sugerindo o delineamento de estudos longitudinais. Ao mesmo tempo, as causas das dificuldades alimentares na primeira infância apontam para uma multiplicidade de variáveis que ainda não são bem conhecidas.

As pesquisas que utilizaram intervenções com os pais mostraram resultados positivos. A partir desta perspectiva, considera-se que é importante ampliar intervenções a fim de dar suporte para que os pais possam expor suas preocupações relacionadas aos problemas alimentares dos filhos e desenvolver mais pesquisas longitudinais com o objetivo de verificar a evolução dos sintomas e também obter dados epidemiológicos.

Os estudos que envolveram os pais trazem à tona a dimensão relacional dos problemas alimentares dos filhos, apontando para a necessidade de compreender o fenômeno deste ponto de vista, isto é, a partir do encontro que se dá entre eles. Assim, sugerem-se estudos que incluam como participantes não apenas as crianças, mas também seus pais e cuidadores.

Também se sugere ampliar os estudos com a metodologia qualitativa a fim de obter maior compreensão do significado que os pais e a família dão para as dificuldades alimentares. Além disso, ressalta-se a importância da Psicologia no desenvolvimento de estudos nacionais, pois assim pode-se ter uma compreensão de como as dificuldades alimentares são abordadas nos diferentes contextos e como esta é percebida pelos pais brasileiros.

\section{Referências}

Adami-Lauand, C. B., \& Ribeiro, R. P. P. (2011). A herança transgeracional nos transtornos alimentares: Algumas reflexões. Psicologia USP, 22(4), 927-942.

American Psychiatric Association. (2013). Diagnostic and statiscal manual of mental disorders ( $5 a$ ed). Arlington, VA, American Psychiatric Publishing.

Ammaniti, M., Lucarelli, L., Cimino, S., D'Olimpio, F., \& Chatoor, I. (2012). Feeding disorders of infancy: A longitudinal study to middle childhood. International Journal of Eating Disorders, 45(2), 272-280.

Atzaba-Poria, N., Meiri, G., Millikovsky, M., Barkai, A., Dunaevsky-Idan, M., \& Yerushalmi, B. (2010). Father-child and 
mother-child interaction in families with a child feeding disorder: The role of paternal involvement. Infant Mental Health J ournal, 31(6), 682-698.

Blissett, J., Meyer, C., \& Haycraft, E. (2011). The role of parenting in the relationship between childhood eating problems and broader behaviour problems. Child: Care, Health and Development, 37(5), 642-648.

Brazelton, T. B. (2002). Momentos decisivos do desenvolvimento infantil. São Paulo, SP: Martins Fontes.

Bryant-Waugh, R., Markham, L., Kreipe, R. E., \& Walsh, B. T. (2010). Feeding and eating disorders in childhood. International J ournal of Eating Disorders, 43(2), 98-111.

Carvalho, A. S. de, Lima, M. C. P., Martins, K. P. H. (2013a). Desnutrição infantil e a relação mãe-bebê: Uma discussão psicanalítica. In Busnel, M. C. \& Melgaço, R. G. (org). O bebê e as palavras: Uma visão transdisciplinar sobre o bebê (pp.261271). São Paulo, SP: Instituto Langage.

Carvalho, Â. S. D., Lima, M. C. P., \& Martins, K. P. H. (2013b). As problemáticas alimentares e a desnutrição na infância: contribuições psicanalíticas. Estilos da Clínica, 18(2), 372-386.

Chatoor, I. (2002). Feeding disorders in infants and toddlers: Diagnosis and treatment. Child and Adolescent Psychiatric Clinics of North America, 11(2), 163-183.

Dovey, T. M., Jordan, C., Aldridge, V. K., \& Martin, C. I. (2013). Screening for feeding disorders. Creating critical values using the behavioural pediatrics feeding assessment scale. Appetite, 69, 108-113.

Farrow, C. V., Galloway, A. T., \& Fraser, K. (2009). Sibling eating behaviours and differential child feeding practices reported by parents. Appetite, 52(2), 307-312.

Forestell, C. A., \& Mennella, J. A. (2012). More than just a pretty face. The relationship between infant's temperament, food acceptance, and mothers' perceptions of their enjoyment of food. Appetite, 58(3), 1136-1142.

Gaffney, K. F., Kitsantas, P., Brito, A., \& Swamidoss, C. S. (2014). Postpartum depression, infant feeding practices, and infant weight gain at six months of age. Journal of Pediatric Health Care, 28(1), 43-50.

Gonçalves, M. J., \& Rodrigues, E. (1998). As perturbações alimentares precoces e a sua avaliação. Análise Psicológica, 16(1), 127-138.

Gusmão, M. H. (2002). Os transtornos e as dificuldades da alimentação. Revista Latinoamericana de Psicopatologia Fundamental, (1), 44-60.

Hoffman, E. R., Hodges, E. A., Propper, C., Postage, P. L., Zipkin, E. C., Bentley, M. E., Ward, D. S., Hamer, R. M., \& Bulik, C. M. 
(2013). Behavioral and psychophysiological responsiveness during child feeding in mothers with histories of eating disorders: A pilot study. Journal of Psychopathology and Behavioral Assessment, 35(4), 578-591.

Jones, C. J., \& Bryant-Waugh, R. (2012). Development and pilot study of a Group skills-and-suport Intervention for mothers of children with feeding problems. Appetite, 58(2), 450-456.

Koh, G. A., Scott, J. A., Woodman, R. J., Kim, S. W., Daniels, L. A., \& Magarey, A. M. (2014). Maternal feeding self-efficacy and fruit and vegetable intakes in infants: Results from the SAIDI study. Appetite 81: 44-51.

Li, R., Scanlon, K. S., May, A., Rose, C., \& Birch, L. (2014). BottleFeeding Practices During Early Infancy and Eating Behaviors at 6 Years of Age. Pediatrics, 134(Supplement 1), S70-S77.

Madeira, I. R., \& Aquino, L. A. D. (2003). Problemas de abordagem difícil: "não come" e "não dorme". J ornal de Pediatria, 79 (Supl. 1), S43-54.

Maldonado-Duran, J. M., Fonagy, P., Helmig, L., Millhuff, C., Moody, C., Rosen, L. \& Van Sickle, G. (2008). In-depth mental health evaluation of a community sample of nonreferred infants with feeding difficulties. International Journal of Eating Disorders, 41: 6, 513-519.

McDermott, B. M., Mamun, A. A., Najman, J. M., Williams, G. M., O'Callaghan, M. J., \& Bor, W. (2010). Longitudinal correlates of the persistence of irregular eating from age 5 to 14 years. Acta Paediatrica, 99(1), 68-71.

McMeekin, S., Jansen, E., Mallan, K., Nicholson, J., Magarey, A., \& Daniels, L. (2013). Associations between infant temperament and early feeding practices. A cross-sectional study of Australian mother-infant dyads from the NOURISH randomised controlled trial. Appetite, 60, 239-245.

Micali, N., Simonoff, E., Stahl, D., \& Treasure, J. (2011). Maternal eating disorders and infant feeding difficulties: Maternal and child mediators in a longitudinal general population study. Journal of Child Psychology and Psychiatry, (7), 800-807.

Nicholls, D., \& Bryant-Waugh, R. (2009). Eating disorders of infancy and childhood: definition, symptomatology, epidemiology, and comorbidity. Child and Adolescent Psychiatric Clinics of North America, 18(1), 17-30.

Östberg, M., \& Hagelin, E. (2011). Feeding and sleeping problems in infancy-a follow-up at early school age. Child: Care, health and development, 37(1), 11-25.

Owen, C., Ziebell, L., Lessard, C., Churcher, E., Bourget, V., \& Villenueve, H. (2012). Interprofessional Group Intervention for Parents of Children Age 3 and Younger With Feeding Difficulties 
Pilot Program Evaluation. Nutrition in Clinical Practice, 27(1), 129-135.

Pinto, E. B. (2004). Os sintomas psicofuncionais e as consultas terapêuticas pais/bebê. Estudos de Psicologia, 9(3), 451-457.

Powell, F. C., Farrow, C. V., \& Meyer, C. (2011). Food avoidance in children. The influence of maternal feeding practices and behaviours. Appetite, 57(3), 683-692.

Reba-Harrelson, L., Von Holle, A., Hamer, R. M., Torgersen, L., Reichborn-Kjennerud, T., \& Bulik, C. M. (2010). Patterns of maternal feeding and child eating associated with eating disorders in the Norwegian Mother and Child Cohort Study (MoBa). Eating behaviors, 11(1), 54-61.

Richter, N., \& Reck, C. (2013). Positive maternal interaction behavior moderates the relation between maternal anxiety and infant regulatory problems. Infant Behavior and Development, 36(4), 498-506.

Rigal, N., Chabanet, C., Issanchou, S., \& Monnery-Patris, S. (2012). Links between maternal feeding practices and children's eating difficulties. Validation of French tools. Appetite, 58(2), 629-637.

Schmid, G., Schreier, A., Meyer, R., \& Wolke, D. (2010). A prospective study on the persistence of infant crying, sleeping and feeding problems and preschool behaviour. Acta Paediatrica, 99(2), 286-290.

Schneider, A., Ramires, V. R. (2007). Primeira Infância Melhor: Uma inovação em política pública. Brasília: UNESCO, Secretaria de Saúde do Estado do Rio Grande do Sul.

Tambelli, R., Odorisio, F., \& Lucarelli, L. (2014). Prenatal and postnatal maternal representations in nonrisk and at-risk parenting: exploring the influences on mother-infant feeding interactions. Infant Mental Health J ournal, 35(4), 376-388.

Thompson, A. L., \& Bentley, M. E. (2013). The critical period of infant feeding for the development of early disparities in obesity. Social Science \& Medicine, 97, 288-296.

Three, Z. T. (2005). Diagnostic classification of mental health and developmental disorders of infancy and early childhood: Revised edition (DC: 0-3R). Zero To Three Press, Washington, DC.

Winnicott, D. W. (1982). A criança e o seu mundo. (6a ed). Rio de Janeiro, RJ : LTC Editora (trabalho original publicado em 1965).

\footnotetext{
Endereço para correspondência

Patrícia Wolff Müller

Universidade do Vale do Rio dos Sinos - UNISINOS

Rua Carlos Barbosa, 80/303-B, Centro, CEP 93800-096, Sapiranga - RS, Brasil

Endereço eletrônico: patyw@terra.com.br
} 


\section{Viviane Salazar}

Universidade do Vale do Rio dos Sinos - UNISINOS

Avenida Unisinos, 950, Cristo Rei, CEP 93022-000, São Leopoldo - RS, Brasil

Endereço eletrônico: viviane.salazar@yahoo.com.br

Tagma Marina Schneider Donelli

Universidade do Vale do Rio dos Sinos - UNISINOS

Avenida Unisinos, 950, Cristo Rei, CEP 93022-000, São Leopoldo - RS, Brasil

Endereço eletrônico: tagmapsi@gmail.com

Recebido em: 29/09/2014

Reformulado em: 11/11/2016

Aceito em: 17/11/2016

\section{Notas}

* Psicóloga, Mestre em Psicologia Clínica pela Universidade do Vale do Rio dos Sinos - UNISINOS/RS.

** Bolsista de iniciação científica PIBIC/CNPq.

*** Psicóloga, Professora do Programa de Pós-Graduação em Psicologia Clínica Universidade do Vale do Rio dos Sinos - UNISINOS/RS. 